\title{
Epilepsy and phenylketonuria: a case description and EEG-fMRI findings
}

\author{
Melania Guida, MDa \\ Ilaria Pesaresi, MD \\ Serena Fabbri, PhD ${ }^{\mathrm{b}, \mathrm{c}}$ \\ Ferdinando Sartucci, MD ${ }^{\mathrm{d}}$ \\ Mirco Cosottini, MD ${ }^{\mathrm{b}, \mathrm{c}}$ \\ Filippo Sean Giorgi, MD, PhDa
}

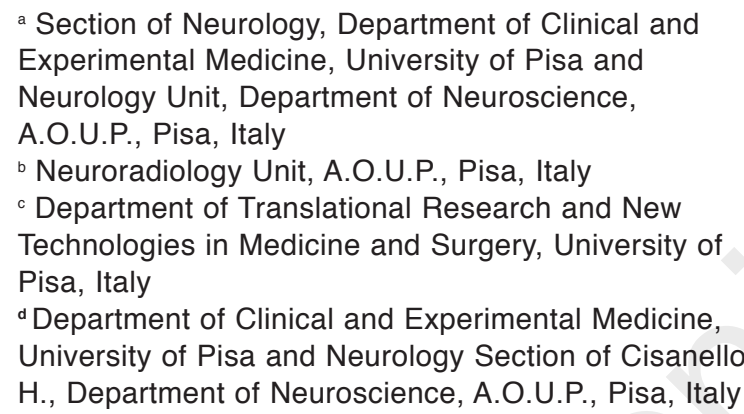

Correspondence to: Filippo Sean Giorgi

E-mail: f.giorgi@ao-pisa.toscana.it

\section{Summary}

Phenylketonuria (PKU) is characterized by phenylalanine accumulation due to phenylalanine hydroxylase deficiency. Up to $50 \%$ of PKU patients experience seizures. We evaluated an adult PKU patient who suffered from absences and primarily generalized tonicclonic seizures, associated with generalized spikeand-wave discharges (GSWs) on EEG. An analysis of blood oxygenation level-dependent (BOLD) signal changes during interictal epileptiform discharges showed early activation of the left perirolandic cortex followed by a BOLD signal decrease within cortical regions belonging to the default mode network and left frontoparietal cortex. Moreover, deactivation of the head of the right caudate nucleus and the left thalamus was observed. The fMRI pattern observed in our patient during GSWs is similar but not identical to that observed in idiopathic generalized epilepsy, suggesting different neurophysiological mechanisms. This is the first description of BOLD-fMRI patterns in a PKU patient with epilepsy. Similar studies in more patients might help to uncover the pathophysiology of seizures in this disease.
KEY WORDS: absences, BOLD, EEG-fMRI, generalized spike-andwaves, phenylketonuria.

\section{Introduction}

Phenylketonuria (PKU) is a metabolic disorder, characterized by accumulation of phenylalanine (Phe) due to phenylalanine hydroxylase $(\mathrm{PAH})$ deficiency. If untreated, it leads to an abnormal phenotype including growth failure, intellectual impairment and seizures. PKU patients may experience different types of seizures, including generalized ones.

We report the blood oxygenation level-dependent functional magnetic resonance imaging (BOLD-fMRI) features recorded during interictal epileptiform discharges (IEDs) in an adult patient affected by PKU, who had experienced absence seizures and primarily generalized tonic-clonic seizures in adolescence and early adulthood, associated with features of idiopathic generalized epilepsy (IGE) on EEG.

\section{Case report}

\section{History and clinical features}

A 45-year-old Caucasian male was admitted as an outpatient to our epilepsy center at the age of 40 . His childhood history showed, from the age of two years, delayed acquisition of walking and language milestones and mild learning difficulties. At the age of 16 years, he experienced a first seizure characterized by eyelid myoclonias and brief psychomotor arrest. In the following months he experienced similar seizures, sometimes triggered by watching television. A year later a generalized tonicclonic seizure occurred. Carbamazepine induced an increase in seizure frequency, and was thus substituted with ethosuximide and primidone, which gave reasonable seizure control. At the age of 18 years, the patient was diagnosed with PKU in a pediatric neurology unit: his blood and urinary Phe levels on admission were $2516 \mu \mathrm{mol} / \mathrm{l}$ and $297 \mathrm{mmol} / \mathrm{l}$, respectively; $\mathrm{PAH}$ gene mutation was confimed. EEG on admission showed prolonged bursts of 3-4 Hz generalized spike-and-wave discharges (GSWs), which increased during 10-15 Hz intermittent light stimulation (ILS) (Fig.1A).

A Phe-poor diet was promptly started, primidone was discontinued and the ongoing ethosuximide was asso- 
ciated with phenobarbital. Within one month cognitive function and seizures had already significantly improved and blood and urinary Phe levels had fallen significantly. Routine EEG had improved too: few GSWs were recorded and were not increased by ILS. Thereafter, the patient experienced absence episodes and sporadic eyelid myoclonias only 2-3 times per year up to the age of 35 years when lamotrigine was associated with phenobarbital and ethosuximide, resulting in complete resolution of absences and eyelid myoclonias up to the present time. EEGs performed annually at our center consistently reveal bursts of 3-4 Hz diffuse sharp waves and GSWs (Fig.1B), not modified by hyperventilation or ILS. A 3T brain MRI scan showed a mild subcortical white matter alteration (Fig.1C).

\section{EEG-fMRI}

The patient underwent an EEG-fMRI investigation performed using a $3 T$ MR scanner. fMRI data were obtained by a $\mathrm{T}^{*}$-weighted gradient recalled echoplanar imaging sequence (TR $2500 \mathrm{msec}$, TE 40 msec, FA $90^{\circ}$, image matrix $128 \times 128$, in plane field of view $220 \times 220 \mathrm{~mm}^{2}$ ) with 28 interleaved slices (slice thickness $5 \mathrm{~mm}$ ) parallel to the anterior-posterior commissural plane, repeated over 300 volumes for a total scanning time of $12 \mathrm{~min} 30 \mathrm{sec}$. The EEG was recorded with an MR-compatible cap as described elsewhere (Pesaresi et al., 2011). Data were analyzed using the FEAT v5.98 tool of the software-package FSL 4.1 .5 (http://www.fmrib.ox.ac.uk/fsl/). The preprocessing of the dataset consisted of spatial smoothing with a $6 \mathrm{~mm}$ Gaussian kernel, temporal filtering with a $100 \mathrm{sec}$ high-pass filter, slice-time correction and motion correction. We explored a time window from 10 sec before until $10 \mathrm{sec}$ after the onset of IEDs, proceeding in $2.5 \mathrm{sec}$ steps. For each step we built up a general linear model (GLM) composed of IED regressor convolved with a Gamma hemodynamic response function (HRF). Motion parameters were also added to the GLM as covariates of no interest. In total, 12 GLMs were computed and statistical maps were obtained with a cluster threshold of $z=2.3$ and applying a $p$-value $=0.05$.

This study was approved by our institutional ethics comittee and the patient gave his written consent to the publication of this case report.

\section{Interictal EEG-fMRI findings}

The resulting statistical maps at the level of the subcortical structures (Fig. 2A) revealed deactivation of the left thalamus and the head of the right caudate nucleus from $5 \mathrm{sec}$ before to $5 \mathrm{sec}$ after onset of IEDs. At cortical levels, statistical maps showed early activation of the left perirolandic cortex (10 sec before IEDs), followed by a BOLD signal decrease in cortical regions belonging to the so-called default mode network (DMN), i.e. the precuneus, lateral parietal cortex, frontomesial cortex and prefrontal cortex, from $5 \mathrm{sec}$ before to $2.5 \mathrm{sec}$ after onset of IEDs. A late BOLD signal decrease was detected in the left frontoparietal cortex (7.5 sec after onset of IEDs) (Fig. 2B).

\section{Discussion}

Phenylketonuria is caused by an inborn error of Phe metabolism (Williams et al., 2008) due to mutation of

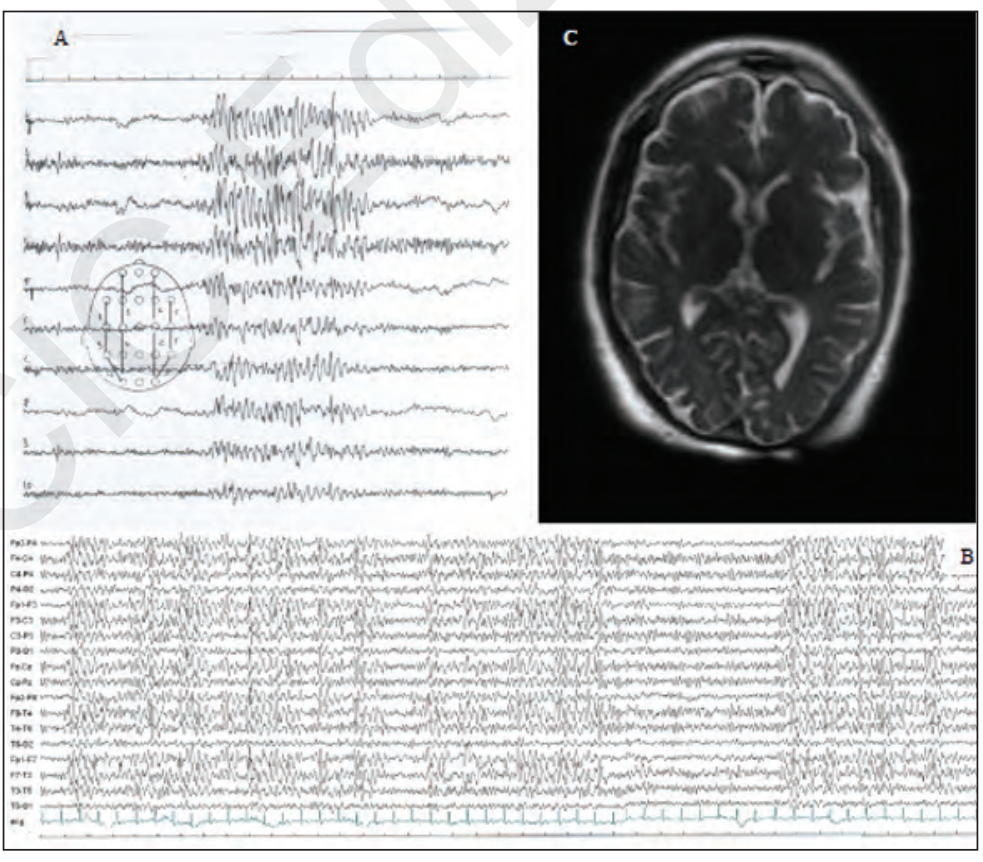

Figure 1 - EEG and MRI features of the patient.

The recording performed at 18 years of age (A) showed a burst of 3-4 Hz GSWs. The EEG pattern during adulthood (B) was characterized by long bursts of 3-4 Hz GSWs and diffuse sharp waves. 3T brain MRI (C) perfomed during adulthood showed mild bilateral subcortical white matter signal alteration. 
the PAH gene located on chromosome $12 \mathrm{q} 23.2$ (Lidsky et al., 1985). Because of the enzyme failure, Phe cannot be transformed into tyrosine (Tyr): the accumulation of toxic byproducts of Phe and the deficiency of Tyr and its downstream products (catecholamines, melanin, thyroxine) together cause the PKU phenotype, which includes growth failure, intellectual impairment and seizures. Several factors contribute to the neurotoxicity occurring in PKU. Brain MRI examinations performed in adult PKU patients invariably show white matter abnormalities (Thompson et al., 1990) and post-mortem brain examinations in PKU patients reveal hypo- or demyelination, independently of a Phe-poor diet (Dyer, 1999). In line with these reports, our patient's brain MRI showed the presence of white matter abnormalities despite the continuation of dietary treatment in adulthood.

There exist no detailed descriptions of the epilepsy/ seizure phenotypes in PKU, although generalized tonic-clonic seizures and myoclonic absences have been suggested to occur in up to $25 \%$ of treated PKU patients (Brenton and Pietz, 2000). Furthermore, EEG abnormalities are frequently observed even in adult patients under a strict dietary treatment (Gross et al., 1981), a finding that suggests a permament alteration. The mechanisms linking Phe levels and seizures are far from clear. However: i) high Phe concentrations are associated with a decrease in brain levels of norepinephrine and serotonin (Puglisi-Allegra et al., 2000), which have anticonvulsant effects (Giorgi et al., 2003); ii) in mice bearing the human $\mathrm{PAH}$ gene mutation a strict interplay between Phe levels and glutamatergic transmission has been observed: due to an inhibitory effect of Phe upon glutamatergic transmission, these mice might have a latent glutamatergic hyperexcitation linked to chronic compensatory upregulation of glutamatergic receptors caused by high pre-treatment Phe levels (Martynyuk et al., 2007). Interestingly, these mutant mice show increased susceptibility to audiogenic seizures, a model of generalized seizures (Martynyuk et al., 2007).

Interictal epileptiform discharges have been present in our patient, in a varying fashion, since a young age: typical GSWs in adolescence/early adulthood and diffuse sharp waves/GSWs in his forties, with disappearence of ILS-triggered bursts.

Our patient's features fit those of an IGE syndrome, since: i) his EEG constantly showed 3-4 Hz GSWs; ii) he reported a history of both absences and generalized tonic-clonic seizures; iii) during his adolescence he experienced ILS-triggered GSWs, which are often described in IGE, especially juvenile myoclonic epilepsy and childhood-juvenile absence epilepsy (CAE-JAE).

The patient also met at least some of the criteria for a diagnosis of eyelid myoclonia with absences (EMA) (Panayiotopoulos, 2005), since he experienced eyelid myoclonia and brief absences found to be related to EEG GSWs and triggered by ILS; however, he never experienced epileptiform discharge during eye closure - this is considered a hallmark of EMA (Panayiotopoulos, 2005) - nor was such discharge mentioned in previous EEG reports.

At least some aspects of the fMRI-BOLD signal modification we observed in our patient are similar to what has been described during GSWs in IGE patients in other EEG-fMRI studies. In particular, deactivation of

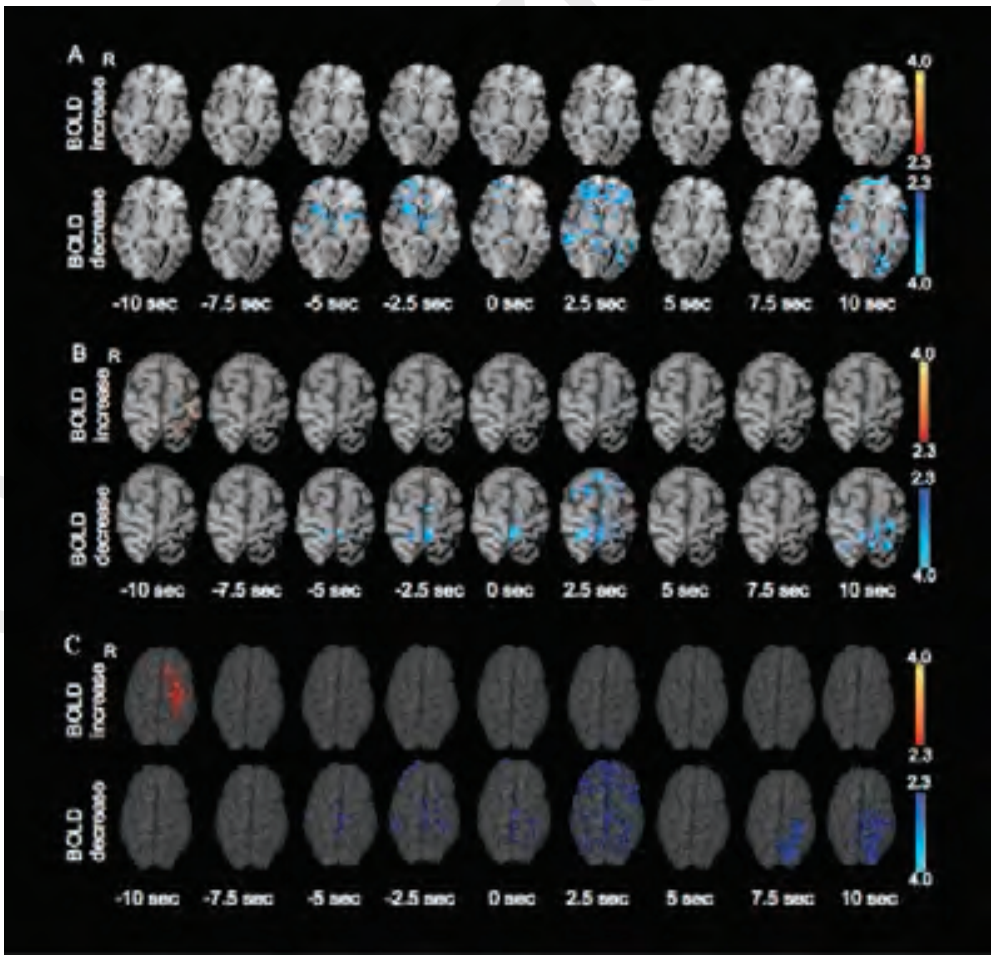

Figure 2 - EEG-fMRI BOLD signal changes during IEDs.

Axial view of fMRI at the level of basal ganglia (A) and at the level of the rolandic knob (B) and a volume rendering of the cortical surface providing a panoramic overview of activation and deactivation (C). Each panel provides a dynamic representation of BOLD activation and deactivation from 10 sec before up to $10 \mathrm{sec}$ after the onset of a GSW burst: the statistical maps obtained by a set of gamma functions with different time peaks are shown. In (A) the BOLD signal changes within the basal ganglia and thalamus are reported. Deactivation of the head of right caudate nucleus and the left thalamus was observed from $5 \mathrm{sec}$ before, until $5 \mathrm{sec}$ after, the onset of IEDs, synchronously with the BOLD signal reduction within the DMN (see below). The BOLD signal changes within the cortex are shown in (B). We observed early activation of the left perirolandic cortex (10 sec prior to onset of IEDs) followed by a BOLD decrease within cortical regions belonging to the DMN (precuneus, lateral parietal cortex, frontomesial cortex and prefrontal cortex) from $5 \mathrm{sec}$ before, until 2.5 seconds after, the onset of GSWs. A late BOLD reduction was detected within the left frontoparietal cortex (from $7.5 \mathrm{sec}$ before to $10 \mathrm{sec}$ after onset of GSWs). Panel (C) shows a volumetric $3 D$ rendering of the brain BOLD-fMR increase/decrease. 
the so-called DMN after onset of GSWs has invariably been described during GSWs in IGE, and particularly in CAE-JAE (Aghakhani et al., 2004; Hamandi et al., 2006; Moeller et al., 2008; Carney et al., 2010; Benuzzi et al., 2012) in which it has been related, theoretically, to the vigilance impairment occurring during prolonged GSWs. Conversely, in many IGE patients, thalamic activation is described at onset of GSWs (Aghakhani et al., 2004; Hamandi et al., 2006; Moeller et al., 2008; Carney et al., 2010; Benuzzi et al., 2012), whereas we detected only an early monolateral thalamic deactivation. Our patient also showed brief unilateral caudate deactivation, similar to that recently reported in a girl with GSWs (Moeller et al., 2010a). Finally, as regards the fMRI findings at cortical level, some studies reported BOLD signal activation in different cortical areas before onset of GSWs (Benuzzi et al., 2012; Moeller et al., 2010b). In a detailed study by Moeller et al. (2010b) in patients with CAE, an early asymmetrical frontal cortical activation was observed in most subjects during absence seizures, similar to what we found in our patient. According to the authors, the difference with other studies describing symmetrical cortical activation in patients with IGE could be related to the different statistical approaches used (individual pattern versus group analysis). In any case, the above findings support the hypothesized role of interplay between the thalamus and the neocortex in the generation of GSWs (Blumenfeld, 2005). Interestingly, a similar left precentral cortex activation during brief diffuse spike-wave discharges has also recently been described (Vaudano et al., 2012) in a patient with reading epilepsy; even though our patient did not show the clinical features of such a localization-related epilepsy, we cannot exclude that, at least in part, these two patients might share some common functional substrates for the generation of IEDs.

Finally, it is interesting to note that BOLD activation of the thalamus and DMN deactivation are constantly observed also in EMA patients, during ictal/interictal GSWs (Liu et al., 2008).

It is worth mentioning some methodological aspects of our study in which, like other authors (Bagshaw et al., 2004; Jacobs et al., 2009; Moeller et al., 2010a), we investigated multiple time intervals from IED onset. However, our study differed from previous ones in that we did not combine different BOLD maps in order to establish the statistically prevalent BOLD phenomenon, but simply reported all the BOLD changes observed in subsequent time intervals, as we were interested in their temporal evolution. Assuming the existence of dynamic processes that evolve over time, we did not compare alternative hypotheses (i.e. alternative HRFs) and did not perform any correction for the number of HRFs employed as they were included in separate GLMs. It is reasonable to run each analysis with the smallest possible $p$ value in order to reduce the total false positive rate of the study. The possibility of false positive results should however be borne in mind when interpreting BOLD statistical maps.

In conclusion, some of the cortical BOLD signal changes observed in our patient during GSWs resem- ble those observed in IGE, and this might be related, at least in part, to the similarity in the interictal EEG patterns between IGE patients and PKU patients with absences.

However, the precise meaning of these fMRI features in IGE, particularly with regard to their pathogenetic implications, is still far from being elucidated.

By the same token, the lack of a thalamic activation, which is a frequent $f M R I$ feature in IGE, suggests that: i) our PKU patient is unlikely also to have IGE as an independent disease;

ii) almost identical electroclinical phenotypes are not necessarily underlain by the same pathogenesis;

iii) the thalamic BOLD activation in IGE may not be as critical for the generation of GSWs as currently believed.

Given the relatively high incidence of PKU among the general population and of seizures among PKU patients, large studies on the characteristics, pathogenesis and possibly fMRI features of the epileptic syndromes occurring in this condition are required to extend our knoweledge of this disease, as well as to disclose new mechanisms in seizure generation.

\section{References}

Aghakhani Y, Bagshaw AP, Bénar GC, et al (2004). fMRI activation during spike and wave discharges in idiopathic generalized epilepsy. Brain 127:1127-1144.

Bagshaw AP, Aghakhani Y, Bénar GC , et al (2004). EEG-fMRI of focal epileptic spikes: analysis with multiple haemodynamic functions and comparison with gadoliniumenhanced MR angiograms. Human Brain Mapping 22:179-192.

Benuzzi F, Mirandola L, Pugnaghi M, et al (2012). Increased cortical BOLD signal anticipates generalized spike and wave discharges in adolescents and adults with idiopathic generalized epilepsies. Epilepsia 53:622-630.

Blumenfeld H (2005). Cellular and network mechanisms of spike-wave seizures. Epilepsia 46 (Suppl 9):21-33.

Brenton DP, Pietz J (2000). Adult care in phenylketonuria and hyperphenylalaninaemia: the relevance of neurological abnormalities. Eur J Pediatr 159 (Suppl 2):S114-120.

Carney PW, Masterton RA, Harvey AS, et al (2010). The core network in absence epilepsy. Differences in cortical and thalamic BOLD response. Neurology 75:904-991.

Dyer CA (1999). Pathophysiology of phenylketonuria. Ment Retard Dev Disabil Res Rev 5:104-112.

Giorgi FS, Ferrucci M, Lazzeri G, et al (2003). A damage to locus coeruleus neurons converts sporadic seizures into self-sustaining limbic status epilepticus. Eur J Neurosci 17:2593-2601.

Gross PT, Berlow S, Schuett VE, et al (1981). EEG in phenylketonuria. Attempt to establish clinical importance of EEG changes. Arch Neurol 38:122-126.

Hamandi K, Salek-Haddadi A, Laufs H, et al (2006). EEG-fMRI of idiopathic and secondarily generalized epilepsies. Neuroimage 31:1700-1710.

Jacobs J, LeVan P, Moeller F, et al (2009). Hemodynamic changes preceding the interictal EEG spike in patients with focal epilepsy investigated using simultaneous EEG-fMRI. Neuroimage 45:1220-1231.

Lidsky AS, Law ML, Morse HG, et al (1985). Regional mapping of the phenylalanine hydroxylase gene and the phenylke- 
tonuria locus in the human genome. Proc Natl Acad Sci USA 82:6221-6225.

Liu Y, Yang T, Liao W, et al (2008). EEG-fMRI study of the ictal and interictal epileptic activity in patients with eyelid myoclonia with absences. Epilepsia 49:2078-2086.

Martynyuk AE, Ucar DA, Yang DD, et al (2007). Epilepsy in phenylketonuria: a complex dependence on serum phenylalanine levels. Epilepsia 48:1143-1150.

Moeller F, Siebner HR, Wolff S, et al (2008). Simultaneous EEG-fMRI in drug-naive children with newly diagnosed absence epilepsy. Epilepsia 49:1510-1519.

Moeller F, Muhle H, Wiegand G, et al (2010a). EEG-fMRI study of generalized spike and wave discharges without transitory cognitive impairment. Epilepsy Behav 18:313-316.

Moeller F, LeVan P, Muhle H, et al (2010b). Absence seizures: individual patterns revealed by EEG-fMRI. Epilepsia 51:2000-2010.

Panayiotopoulos CP (2005). Syndromes of idiopathic general- ized epilepsies not recognized by the International League Against Epilepsy. Epilepsia 46(Suppl 9):57-66.

Pesaresi I, Cosottini M, Belmonte G, et al (2011). Reproducibility of BOLD localization of interictal activity in patients with focal epilepsy: intrasession and intersession comparisons. MAGMA 24:285-296.

Puglisi-Allegra S, Cabib S, Pascucci T, et al (2000). Dramatic brain aminergic deficit in a genetic mouse model of phenylketonuria. Neuroreport 11:1361-1364.

Thompson AJ, Smith I, Brenton D, et al (1990). Neurological deterioration in young adults with phenylketonuria. Lancet 336:602-605.

Vaudano AE, Carmichael DW, Salek-Haddadi A, et al (2012). Networks involved in seizure initiation. A reading epilepsy case studied with EEG-fMRI and MEG. Neurology 79:249-253.

Williams RA, Mamotte CD, Burnett JR (2008). Phenylketonuria: an inborn error of phenylalanine metabolism. Clin Biochem Rev 29:31-41. 